\title{
Langage et travail : \\ une dynamique de « double anticipation » pour la formation professionnelle
}

Louis Durrive*

\section{Résumé}

En formation professionnelle, la question se pose du retour d'expérience. Qu'est-ce que nous apprend le travail, non seulement en termes de savoirs à mobiliser dans l'action, mais aussi dans la gestion des épreuves au moment d'agir, quand il faut tenir compte de ce qui vient d'arriver et de ce qui s'annonce, des initiatives que les autres sont en train de prendre, des enjeux de l'action collective, des risques que l'on doit soi-même assumer en responsabilité ?Pour rendre compte de toute cette richesse, il faut comprendre pourquoi il est si difficile de "dire son travail " et imaginer un dispositif capable d'inciter chacun à se prêter à l'exercice. C'est le double objectif du présent article.

Mots-clés : Récit d'activité. Formation professionnelle. Langage et ergologie. Analyse de l'activité.

\section{Introduction}

Faire le récit de son travail n'est pas une chose simple. Tout se passe comme s'il y avait une sorte de contradiction entre vivre et dire, chaque fois que l'on est invité à parler de son expérience industrieuse. Le langage nous tire vers des réalités déjà connues tandis que le travail nous entraîne vers des situations inédites, très spécifiques. Nous ne disposons que d'un vocabulaire banal pour rendre compte d'un moment de vie complexe. Difficile dans ces conditions d'exprimer quelque chose de personnel à propos de l'activité productive. Travailler reviendrait, semble-t-il, à reproduire ce que l'on sait déjà et que l'on nous a commandé de faire et refaire, sans au-

Professeur en sciences de l'éducation, Université de Strasbourg/Espe (France) - Laboratoire LISEC.

Data de submissão: ago. 2019 - Data de aceite: out. 2019 http://dx.doi.org/10.5335/rdes.v15i3.9721 
cune place pour la créativité, l'initiative, l'imagination, l'engagement de soi.

En formation professionnelle, cette relation difficile entre le langage et le travail nous fait perdre le bénéfice d'une grande partie de l'expérience en entreprise. Au retour d'une période de production, l'apprenti a bien souvent l'impression de n'avoir rien à dire d'intéressant alors qu'il a été absorbé durant plusieurs jours par des tâches qui mobilisaient toutes ses ressources intellectuelles, psychiques, physiques. C'est ce déficit dans les retours d'expérience qui nous a conduit à réfléchir à un dispositif pour apprendre à dire son travail sous la forme d'un récit d'activité, dans une perspective ergologique.

\section{Le travail approché par les deux usages du langage}

L'activité symbolique accompagne toute confrontation au monde réel, si bien que l'activité humaine a toujours une double dimension, historique et langagière. Cependant l'expérience ne prend pas la même allure selon le rôle que joue le médium qu'est le langage. S'il s'agit d'un vécu singulier, le langage sera de proximité : c'est l'expérience au sens de l'épreuve du monde, dans une situation toujours unique, un «ici et maintenant ». S'il s'agit de retenir une leçon de la vie, le langage sera au contraire distancié : c'est l'expérience comme connaissance du monde ou expérience acquise. Dans ce dernier cas ("Erfahrung " en allemand), les mots de la langue seront non circonstanciels, génériques, conceptuels. A l'inverse dans le cas précédent («Erlebnis » en allemand), les mots parfois tordus ou détournés et leur agencement syntaxique seront noués aux circonstances selon un référentiel qui risque d'échapper à ceux qui ne connaissent pas la réalité très locale. L'activité symbolique est par conséquent hautement flexible. C'est que le langage n'est pas à proprement parler un outil que l'on pourrait utiliser à un moment donné pour le déposer ensuite ; il est constitutif du milieu dans lequel on se trouve immergé. En tant que médium de notre rencontre avec le monde, il se prête à un usage spontané ou bien très volontaire, familier ou bien académique, plutôt ouvert ou plutôt fermé. On reconnaît ainsi le «continuum formel / informel » qui caractérise le langage (SCHWARTZ, 1992, p. 79).

Cette double caractéristique du langage nous apparaît d'une importance cruciale en formation professionnelle. Le langage peut révéler et masquer en même temps, faire découvrir un aspect de la réalité et en cacher un autre simultanément. Dans la mesure où il porte vers le général, le langage offre la possibilité d'établir des catégories, d'identifier et désigner les choses mal- 
gré l'infinie diversité des situations rencontrées. C'est l'étape qui précède la hiérarchisation, l'attribution d'une valeur (évaluation) à ce que l'on sait désormais appeler par son nom. En effet après avoir séparé grâce aux mots adéquats le donné immédiat, on devra y reconnaître un ordre, une cohérence et cela avant de pouvoir apprécier ses propres marges d'intervention pour transformer l'existant. C'est ainsi que (comme le rappelle Y. Schwartz, cf. infra) "qualifier» quelque chose veut dire à la fois nommer et normer, distinguer et ordonner - séparer d'abord pour réunir ensuite. Tous les apprentissages en formation professionnelle se fondent sur ce travail linguistique comme première approche du métier.

Cependant en procédant par catégorisation, le langage nous éloigne inévitablement du moment unique, historique - le nœud de circonstances dans lequel nous agissons, un moment qui est toujours un présent complexe, constitué des trois temps : un présent $d u$ passé, un présent du présent, un présent du futur, selon l'expression augustinienne commentée par Ricœur (1983, p. 28). Lorsqu'il cherche à décrire une situation de travail, le langage formel ne retient dans ses filets que les données générales, la déclinaison de la situation-type. Tout en permettant de reconnaître et de faire reconnaître le travail en cours, le langage masque alors par son découpage à grands traits de nombreuses contraintes liées aux conditions présentes de l'action qui est engagée. Ce qui reste dans l'ombre, ce ne sont pas des détails au sens d'une information accessoire, sans importance pour la conduite des opérations. La plupart du temps, ce sont au contraire autant de micro-défis à surmonter, qui vont obliger les protagonistes de la situation de travail à repenser plus ou moins leurs manières de s'y prendre.

La distinction entre les deux usages de la langue est bien connue des grammairiens qui distinguent les énoncés ancrés et les énoncés coupés. Le message est dit coupé lorsqu'il est produit indépendamment de la situation de référence, tandis qu'il est dit ancré lorsque l'acte de sa production est intimement lié aux circonstances. Dans l'ancrage d'un ici-maintenant (hier, aujourd'hui ou demain), ce sera le discours direct, la conversation entre un «je » et un « tu».A l'opposé, dans un lieu et un temps coupé (la veille, ce jour-là, le lendemain), le discours sera indirect, à distance, tel qu'on le trouve par exemple dans un rapport de stage ou dans le compte-rendu d'une opération.

En formation professionnelle, lorsqu'il s'agit de rendre compte de l'activité de travail, on ne tire pas parti de cette distinction des grammairiens, pourtant fondamentale à nos yeux. Nous pouvons le regretter dans la mesure où - comme 
nous voudrions le montrer dans la suite de cet article - les deux usages linguistiques dont nous parlons correspondent à deux éclairages complémentaires sur une même réalité, à savoir l'activité humaine : l'activité telle qu'elle est repérable extérieurement, donc observable (" avoir une activité ») et l'activité telle qu'elle se manifeste intérieurement, à travers les débats du sujet en train de faire quelque chose («être en activité »). La raison pour laquelle la distinction " coupé-ancré » nous semble particulièrement pertinente en formation professionnelle, est la suivante. Toutes les questions liées au travail et à son corollaire qui est la compétence sont à aborder en prenant en compte la tension entre le registre du général et celui du spécifique : or faire la différence entre un énoncé coupé et un énoncé ancré, ce n'est pas seulement distinguer deux manières de parler, c'est aussi - nous venons de le dire - reconnaître deux façons de se rapporter à la réalité, y compris dans la situation à vivre. Pour reprendre le vocabulaire ergologique, c'est la distinction entre le registre de la désadhérence et celui de l'adhérence.

L'adhérence est le terme choisi par le philosophe Yves Schwartz pour décrire le défi de vivre au plus près des contraintes réelles, afin de tenter de les utiliser comme des opportunités : «il n'y a aucune vie humaine qui ne soit comme appelée à vivre, pour une part, dans ce que nous nommons "l'adhérence" : soit la mobilisation de nos énergies, incorporées dans nos facultés intellectuelles comme dans nos équipements biologiques, pour détecter ce qui fait point de résistance et point d'appui dans le présent du milieu à vivre » (SCHWARTZ, 2009, p. 16).

La désadhérence, c'est

[...] la capacité d'inventer un mode de se mouvoir - ce sera la pensée humaine - qui soit à distance, déconnectée plus ou moins profondément de la situation immédiate, de ses sollicitations, de ses urgences. Cela permettra, dans le long terme, de produire d'abord le langage articulé, mais dans la continuité, le concept, jusqu'à son pôle extrême, le concept scientifique. [...] Le fait de penser à distance, donc de catégoriser, c'est d'une certaine manière, une invention de norme [...]. Mais catégoriser anticipativement des éléments du monde, c'est aussi les "qualifier" [...]. On subsume un cas sous un concept - voir la qualification comme acte juridique - mais on ne peut pas ne pas en même temps créer une relation qualitative, polarisée, en valeur, avec ce qu'on vise (SCHWARTZ ; DURRIVE, 2009, p. 62).

On le voit, la distinction introduite par Y. Schwartz entre l'adhérence et la désadhérence va encore plus loin que la reconnaissance de deux modalités d'approche de la réalité humaine. C'est un binôme dont l'ambition est de décrire l'activité comme un va-et-vient entre une immersion dans l'expérience du présent et une distanciation permise par le langage.

Agir, pour un être humain, ce sera tenter d'intervenir sur le monde selon les configurations du moment présent - 
c'est l'adhérence - au nom d'un projet qui est conçu par soi ou par d'autres et en dehors de l'actuel : c'est la désadhérence. Une illustration de ce va-et-vient est le fameux écart entre le prescrit et le réel, repéré par l'ergonomie francophone dès les années 1950. De son côté, l'approche ergologique va prolonger cette analyse à partir des années 1980 en cherchant à comprendre la gestion de cet écart (donc le va-et-vient) afin de mieux interpréter l'activité du travailleur.

Si l'analyse du travail peut considérer que le producteur est toujours placé dans une tension entre le registre du général et celui du spécifique, il pourra en être de même dans l'approche du phénomène de la compétence. Ce qui est répertorié dans un référentiel sous le nom de compétence, c'est une manière de parler de l'activité humaine dans ce qu'on appelle une famille de situations. Aussitôt nous reconnaissons le paradoxe du discours sur la compétence : prétendre parler sur le mode général d'un phénomène très singulier, une «rencontre de rencontres » (SCHWARTZ ; DURRIVE, 2009, p. 225), c'est-à-dire une situation de travail issue d'un croisement d'histoires singulières, donc forcément spécifique. Comme on le sait, la connaissance se distingue de la compétence avec le passage par l'action, autrement dit l'épreuve du réel, de la situation spécifique «ici et maintenant». Et en même temps, il n'est pas question de s'enfermer dans la singularité : tout l'intérêt du discours sur la compétence est bien de pouvoir dire quelque chose de général, qui autorise l'anticipation. On retrouve là l'intérêt de mobiliser en formation professionnelle les deux usages du langage : ancré et coupé ; en adhérence et en désadhérence. Ce double éclairage sur la réalité humaine est précieux aussi bien pour évaluer que pour former ou encore encadrer le travail, comme nous allons le voir par la suite avec la description de notre dispositif pour faire « dire le travail ».

\section{Langage et renormalisation}

Nous allons encore apporter quelques éléments de réflexion théorique avant de présenter plus précisément notre dispositif. Le discours « sur» le travail correspond à un usage réfléchi de la langue, tandis que le discours «dans » le travail (l'échange plus ou moins improvisé dans l'action) est associé à un usage spontané de celle-ci. Le langage formel est dominant, puisque c'est à travers lui que sont fixés les consignes et modes opératoires. Chez les gens de métier, c'est le langage légitime, celui qui fournit les termes adéquats pour décrire le travail. De son côté le langage en situation, celui de l'activité industrieuse, n'est pas une version appauvrie ou dégradée de la norme langagière du métier concerné. C'est plutôt un usage ouvert de la langue : 
à l'intérieur des contraintes du travail, l'être d'activité cherche à reprendre l'initiative, à " renormaliser » (dans le vocabulaire ergologique), et de la même façon qu'il procède pour ne pas se laisser entièrement contraindre par le milieu, il va résister aux contraintes de la norme langagière et adopter des ressources symboliques quelque peu différentes, qu'il juge plus adaptées à ce qu'il est en train de faire, plus conformes aussi à ce qu'il perçoit en termes d'enjeux. Ce ne sont pas deux discours parallèles dans un même milieu de travail, mais c'est une activité symbolique qui prend appui sur deux manières de penser et de gérer la confrontation au monde réel : à distance et en proximité. L'activité langagière trouve sa dynamique dans la relation dialectique entre le formel et l'informel, le premier facilitant l'organisation et le second la mise en œuvre du programme de travail. Il faut cependant ajouter que si le langage est une activité dans le sens développé par le linguiste Daniel Faïta (2003, p. 177), il se présente comme une activité dans l'activité. Le jeu dialectique est en effet reproduit dans la relation entre le langage et le travail. L'organisation d'une situation productive est rendue possible par le langage " figeant provisoirement l'historique " à travers les normes antécédentes (SCHWARTZ, 1992, p. 77), ce qui correspond à une première forme d'anticipation. Cependant l'histoire continue, les contraintes conjoncturelles ne cessent de resurgir et obligent à reformuler ce qui a été prévu, à le repenser localement afin d'être efficace : c'est une deuxième forme d'anticipation, dans la mesure où la vie réelle a repris son antériorité par rapport à ce qui a été conceptualisé, pensé à l'avance. À ce moment-là, la norme langagière " ne peut être qu'un moment, particulièrement instable, $d u$ rapport entre symbolisme et activité réelle $»$ (Ibid.).

On parlera de "double anticipation " pour décrire en quelque sorte la course-poursuite du concept et de la vie, toujours portée par le médium de la langue : grâce au langage conceptuel, on dispose des moyens de penser le pensable "en désadhérence ", d'imaginer et d'organiser à l'avance des situations à vivre ; grâce au langage en situation, historique, on parvient à gérer " en adhérence » l'expérience de la réalité, qui toujours déborde ce qu'on a pu conceptualiser, avec la vie qui charrie les singularités parfois les plus surprenantes. Puis, de nouveau, ce qu'on peut appeler l'effort de connaître cherchera à conquérir sa capacité à anticiper les situations à venir, tandis qu'il sera à son tour dépassé par la vie, autrement dit par l'effort de vivre qui incite tout être d'activité à réaménager le plan de travail en fonction des configurations du moment. 
Nous avons vu la dialectique interne à l'activité symbolique (formel et informel) ainsi qu'une dialectique plus large propre à l'activité normative, celle du langage et du travail. C'est à chaque fois une sorte de contradiction féconde, le concept cherchant à dépasser la vie afin de mieux la contrôler mais indéfiniment débordé par elle, par le surgissement de l'inattendu. C'est là, dans cette contradiction, que nous pouvons comprendre l'origine de notre difficulté à rendre compte de l'expérience industrieuse, autrement dit à gérer la rencontre du langage et du travail.

\section{L'obstacle de « l'impossible »}

Le premier obstacle à surmonter lorsqu'il s'agit de "dire le travail ", c'est d'accepter de ne pas en rester à notre perception globale des choses. «Pensée et langage, originellement destinés à organiser le travail des hommes dans l'espace, sont d'essence intellectuelle » fait remarquer Bergson (2003 : 87). Nous opérons des différences dans notre environnement uniquement à grands traits, en fonction de ce qui nous semble utile de retenir et sans aller y voir de près. Et le langage ne fait qu'accentuer cette tendance, puisque les mots sont génériques. Notre perception est utilitaire, guidée par le besoin : or, « le besoin va droit à la ressemblance ou à la qualité et n'a que faire des différences individuelles » (BERGSON, 2013, p. 172), le souci du détail étant «un luxe de la perception » (ibid.).

L'auteur nous explique que les origines du mot et de l'idée sont à chercher dans une préoccupation humaine de retrouver la même propriété quand les choses sont diverses, de se les représenter de la même manière, d'opérer des groupements qui suggèrent le même parti à tirer, la même action à réaliser. «Les choses que le langage décrit ont été découpées dans le réel par la perception humaine en vue du travail humain » écrit Bergson (2003, p. 87). Il considère en effet que la fonction primitive du langage est sociale, liée à l'acte de travail, c'est-à-dire à la nécessité (immédiate ou à venir) de communiquer et de coopérer, transmettre des ordres et des avertissements afin de fabriquer ensemble, vendre, attaquer ou se défendre.

Si nous considérons le point de vue de l'organisateur du travail, nous ne sommes pas étonnés de constater sa tendance à étiqueter les choses, à parler des situations productives dans les grandes lignes. Sa position sociale l'éloigne d'emblée des réalités du moment de l'action. En revanche, nous pouvons être davantage surpris de faire le même constat du point de vue des travailleurs, ceux qui conduisent les opérations sur le terrain. Eux aussi vont mobiliser les grandes catégories du langage pour décrire leur 
quotidien : ils parleront le plus souvent des tâches et de leur déroulement, et ils seront même réticents à donner des précisions, à entrer dans les détails. Ils se diront convaincus que c'est toujours la même chose, que leur travail est essentiellement une routine. Cela rejoint l'idée que notre représentation du travail et notre manière d'en parler se placent toutes deux dans la généralité.

Or, qu'avons-nous appris des travaux en analyse du travail et en particulier de l'ergonomie et l'ergologie ? Nous avons eu au fil des années la démonstration scientifique de ce que Canguilhem appelle "l'infidélité du milieu» (2003, p. 130) : jamais une situation de vie ne se reproduit à l'identique car il est impossible d'inventorier en amont l'ensemble des contraintes, de repérer d'avance tout ce qu'il faut prendre en compte au moment d'agir. Dit autrement, on ne peut espérer entreprendre un travail sans voir surgir de nouvelles contraintes générées par une réalité mouvante. Toute mise en œuvre d'un programme de transformation du milieu suppose une actualisation de ce qui a constitué le cadre de l'action. Car l'approche par l'organisation est toujours une généralisation, donc une forme d'abstraction, tandis que l'expérience du travail est une confrontation aux faits eux-mêmes. La généralisation n'appréhende pas directement les faits, il n'existe pas de « fait général »- une expression que Canguilhem tient pour une "monstruosité logique " (2006, p. 63) dans la mesure où le fait est forcément particulier et la généralisation est nécessairement l'aboutissement d'un processus d'interprétation.

Dans ce cas, pourquoi les travailleurs (nous pensons aussi bien aux salariés qu'aux apprentis) ne sont-ils pas enclins à rapporter eux-mêmes le détail des faits lorsqu'on les invite à dire ce qu'ils font? Pourquoi s'enferment-ils le plus souvent dans les catégories du langage qui ignorent la singularité des situations? Parce que les représentations et les mots qui les véhiculent nous renvoient au registre de l'intellectuel, comme nous l'avons vu avec Bergson - et cela ne suffit pas à faire le tour de la réalité du travail. Travailler, c'est aussi faire une expérience de vivant, traverser une épreuve pour toute la personne, physique aussi bien que psychique, c'est-à-dire « un corps-soi » dans les termes d'Yves Schwartz (2000, p. 664). Il y a par conséquent un écart entre ce que les travailleurs vivent et ce qu'ils disent. Faire advenir l'expérience vécue dans le registre du langage, ce n'est pas simple et cela suppose une démarche volontaire et organisée.

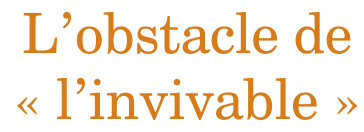

Le second obstacle à surmonter au moment de "dire le travail », c'est d'oser entrer en scène en tant que sujet de ses 
normes, non seulement acteur mais encore auteur de ce que l'on entreprend.

Comme Yves Schwartz le rappelle dans sa définition de la désadhérence (cf. supra), l'effort de penser à distance et de catégoriser conduit à inventer une norme, un « devoir-être ». Or la société dans laquelle évolue l'individu le précède et l'immerge dans un bain de normes, avec un langage qui se veut coercitif : «Le langage est une législation, la langue en est le code. Nous ne voyons pas le pouvoir qui est dans la langue, parce que nous oublions que toute langue est un classement et que tout classement est oppressif : ordo veut dire à la fois répartition et commination " nous explique Roland Barthes (1978, p. 12). Voilà donc une nouvelle difficulté dans la mobilisation du langage pour découvrir le travail. Après avoir compris que ce médium relaie une perception globalisante, généralisante, qui nous rend aveugle aux contraintes, nous devons nous rendre compte qu'il est aussi un relais de pouvoir. Le langage nous contraint, nous détermine dans nos intentions et nos manières de communiquer. À travers ses règles de fonctionnement, le langage force à dire certaines choses de soi, jusqu'à les penser à propos de soi-même.

S'agissant dans notre cas de la formation professionnelle, nous sommes avertis que le système d'éducation est tout entier pris dans les filets du politique. "Qu'est-ce, après tout, qu'un sys- tème d'enseignement ", nous dit Michel Foucault, "sinon une ritualisation de la parole ; sinon une qualification et une fixation des rôles pour les sujets parlants [...] ; sinon une distribution et une appropriation du discours avec ses pouvoirs et ses savoirs ?» (1971, p. 46-47). Tout système institutionnel constitue ainsi un système d'assujettissement par le discours, dans la perspective structuraliste.

Là encore l'analyse de l'activité de travail - celle qui s'inspire de Georges Canguilhem - nous permet de ne pas forcément contredire l'approche que l'on vient d'évoquer mais de prendre en compte sa part de vérité et de chercher à aller plus loin. De quoi s'agit-il en effet? Il s'agit pour les individus au travail (et en formation) d'entrer dans un milieu déjà structuré : une entreprise, un centre de formation, ou toute autre institution. La structure d'accueil a le projet d'obtenir la conformité des manières de penser et d'agir de ses membres et pour cela, elle déploie toute la puissance de son cadre normatif. Ce qui est visé, c'est l'assujettissement, la soumission d'un «sujet » au sens passif du terme. L'individu étant relativement influençable, il va être perméable aux exigences extérieures et va faire l'effort de reconnaître la contrainte et de s'y soumettre : c'est le processus de subjectivation qui débouche sur une appropriation de la norme (normativité). Toutefois, comme nous l'avons appris de Canguilhem, 
l'histoire ne s'arrête pas là. Le processus de réception de la norme se poursuit au plus intime des débats du sujet : celui-ci, au nom d'une revendication existentielle de centralité, va chercher à reprendre une forme d'initiative. Il ne se laisse pas réduire à un "carrefour d'influences " (CANGUILHEM, 2006, p. 197) et va en quelque sorte " contester » ce qu'on prétend faire de lui, chercher autant que possible à orienter l'usage de soi par soi - et non pas uniquement par les autres. C'est en fin de compte un repositionnement (préférer telle manière de faire par rapport à toutes les options possibles) qui sera à l'origine d'un comportement conforme : le processus de subjectivation se prolonge ainsi jusqu'à rendre le sujet à nouveau actif, capable de piloter la contrainte (celle que l'on a cherché à lui imposer) afin de ne pas s'aliéner, de ne pas perdre la conduite de sa propre existence. "Tout homme veut être sujet de ses normes » écrira Canguilhem (1947, p. 135). La normativité ici s'entend alors dans un sens positif, correspondant à un processus de subjectivation active - et la conformité est obtenue à l'issue non pas d'une coercition mais d'un « débat de normes » (SCHWARTZ; Durrive, 2009, p. 254) suivi d'un essai de « renormalisation » (2009, p. 259) - un essai, car jamais joué d'avance (autrement dit, la norme peut échouer à faire autocontrainte et agir uniquement par contrainte).
Tout ce que nous venons de dire se comprend dans le prolongement du point précédent, celui qui présente le caractère « impossible» d'une standardisation des situations de travail : il est impossible de se contenter d'une approche générale, conceptuelle, de la vie réelle dans la mesure où tout moment à vivre voit surgir de nouvelles contraintes. Qu'il soit apprenti ou professionnel chevronné, tout travailleur doit relever le défi de l'incertitude au moment d'agir, parce qu'aucune situation ne sera entièrement pensée à l'avance, standardisée.

Qu'est-ce qui pousse à relever le défi, à reprendre l'initiative face aux contraintes - qu'elles soient ou non anticipées ? Il y a deux raisons à cela. C'est d'abord la reconfiguration du milieu, toujours " infidèle ", toujours mouvant, variable ; cette instabilité (en référence à ce que l'ergologie appelle «l'impossible ») oblige en effet à repenser localement et dans un souci d'efficacité ce qui a été pensé à distance, antérieurement et abstraitement. C'est ensuite et plus profondément parce que d'un point de vue anthropologique, l'être humain ne peut et ne veut pas vivre son existence à travers le point de vue d'autrui : c'est cela l'exigence de centralité, la revendication de singularité (celle précisément que l'on brutalise dans les cas de travail aliéné). Yves Schwartz présente cette exigence comme une nouvelle butée : "l'invivable ", au sens où il serait invi- 
vable pour l'humain d'agir en étant privé d'initiative (SCHWARTZ, 2000, p. 612).

C'est ainsi que l'on comprend le couple "impossible-invivable " : impossible d'anticiper toutes les contraintes, puisqu'elles rebondissent toujours ; et ce même phénomène nourrit l'invivable, c'est-à-dire l'initiative qui rebondit toujours elle aussi.

« Jamais un ouvrier ne reste devant sa machine en disant : je fais ce qu'on me dit » : cette affirmation qu'Y. Schwartz a retenue du discours d'un travailleur (SCHWARTZ, 2012, p. 21) contient quelque chose de très fort puisqu'elle nous rappelle que fondamentalement l'humain ne se contente pas d'exécuter tel un automate. Et voilà comment l'histoire humaine s'écrit, car si l'initiative ne rebondissait pas face aux contraintes, nous serions enfermés dans une reproduction stérile et sans avenir. Au contraire, la dialectique des contraintes et des initiatives nous fait entrer dans une dynamique de "double anticipation ". Chacun est confronté à des contraintes (des normes antécédentes) et va chercher à exister face à elles, à « renormaliser ». Il construit par là son individualité, il développe sa subjectivité. C'est l'étape de la personnalisation, autrement dit la manière dont la personne au travail va gérer personnellement l'écart entre le prescrit et le réel (impossible/ invivable).

Comme le fait remarquer Y. Schwartz, « il n'y aurait pas de problème avec l'activité, cette invitée féconde et encombrante, si la rationalité des normes qui anticipent tout agir social dissipait ou rendait inutile, pour l'essentiel, l'obligation faite à chacun de repenser dans son intime, ses choix d'être et de vie. Obligation faite de "penser ", donc de produire, d'une manière ou d'une autre, du savoir. Obligation faite de " choisir ", donc de se situer, d'une manière ou d'une autre, dans un monde de valeurs. » (SCHWARTZ ; DURRIVE, 2009, p. 221-222). En travaillant, chacun produit et « se produit " au sens où il se construit comme sujet, être d'initiative et d'évaluation.

\section{Un dispositif pour gérer la rencontre problématique du langage et du travail}

Résumons-nous. Nous avons d'abord reconnu deux usages du langage, un usage informel et un usage formel. Le langage de proximité correspond à l'expérience vécue, tandis que le langage distancié convient à l'expérience réfléchie. On retrouve chez les grammairiens une attention à ces deux manières de parler, avec l'énoncé ancré ou coupé. Au-delà de l'énoncé cependant, ce sont deux façons différentes de se rapporter à la réalité : en adhérence ou en désadhérence. Une première approche colle à la situation de vie, tandis qu'une seconde cherche à décoller de celle-ci grâce à la force du concept. Toutefois les deux approches ne 
sont pas simplement juxtaposées, elles entretiennent une relation dialectique puisque chacune cherche à dépasser l'autre en prenant appui sur elle. C'est la « double anticipation ", l'approche conceptuelle visant à contrôler la situation à vivre, tandis que la situation à vivre profite du cadre conceptuel pour faire surgir de l'inattendu. En pointillés, on reconnaît là la relation entre le langage et le travail car le premier est le support du concept tandis que le second s'entend ici comme une expérience de vie. Ce sont finalement ces caractéristiques de la relation entre le langage et le travail qui vont nous permettre de comprendre pourquoi il peut être si difficile de «dire le travail».

Une première difficulté est de se heurter à l'obstacle de "l'impossible ". Le langage a tendance à nous élever à un niveau de généralité qui masque la complexité du moment de vie unique que l'on voudrait rapporter. C'est pourquoi il y a toujours un écart entre ce que l'on dit et ce que l'on vit, particulièrement à propos du travail. Mais cela entraîne une autre difficulté, qui est l'obstacle de «l'invivable». Ignorer qu'il est impossible d'éviter les contraintes non anticipées, soit liées aux circonstances du moment d'agir, c'est aussi ignorer qu'il serait invivable pour l'humain d'être privé d'initiative. De même que les contraintes inattendues sont toujours là, de même l'initiative qui va les gérer est toujours là. Or le langage, dont la caractéristique est d'être à la fois globalisant et dominant, bute contre cet impossible-invivable. Il ne donne pas de visibilité à notre rencontre forcément complexe avec le réel ni a fortiori à nos efforts pour relever le défi et nous construire comme sujets actifs. C'est pourquoi le langage n'offre pas immédiatement une caisse de résonance à la subjectivité dans le travail.

Il importe à présent de réfléchir aux implications de notre analyse. Quel dispositif peut-on mettre en place pour rendre compte le plus fidèlement possible de l'expérience du travail ? Celle-ci se présente comme une renormalisation permanente, entendue comme une actualisation / personnalisation des normes antécédentes. Restituer son travail, cela signifie rendre accessibles en les verbalisant la particularité de la situation (sous l'angle de l'actualisation des normes) et la singularité de la réponse apportée par celui qui agit (sous l'angle de la personnalisation des normes).

\section{Une première phase du dispositif pour dire le travail}

Le dispositif que nous allons décrire est directement inspiré de l'approche ergologique de l'activité humaine. Nous avons repéré deux obstacles dans notre 
projet de faire « dire le travail » : l'impossible et l'invivable. Surmonter le premier suppose d'inciter le narrateur à mettre en mots l'écart à la norme (davantage que l'écart au prescrit dans la mesure où la consigne n'est qu'un aspect du cadre normatif, néanmoins important). Franchir le second obstacle, c'est lui donner des raisons de rendre compte de son engagement dans l'action, de l'effort qu'il manifeste pour gérer cet écart et reprendre l'initiative - ce qui veut dire réaliser le travail demandé au prix d'un usage de soi, d'une mobilisation de la subjectivité.

Ce double objectif constitue la première étape de notre dispositif pour réaliser un récit d'activité. Le résultat attendu est le dépliage de ce qu'Yves Schwartz nomme les triangles "agir, savoir, valeurs " (SCHWARTZ, 2015, p. 132). En effet dans le registre de l'organisation que nous reconnaissons comme la première anticipation (la pensée conceptuelle va imaginer une situation de vie et la préparer), nous allons faire l'inventaire des actions demandées et des savoirs qui leur sont associés. Mais nous avons compris désormais que le couple « agir + savoirs » ne résume pas à lui seul le travail, puisqu'en deuxième anticipation (là où opère la pensée agissante), la mise en œuvre du programme n'évitera pas le jaillissement de l'impré$\mathrm{vu}$, donc de contraintes nouvelles en lien avec les micro-événements. Comme le dit
Y. Schwartz dans l'extrait précédemment cité, le travailleur se trouve inévitablement dans "l'obligation de penser ", de réfléchir au plan logique (et là, il peut disposer des savoirs), mais aussi et surtout dans "l'obligation de choisir ", ce qui veut dire de se placer au plan axiologique, là où les savoirs ne suffisent plus. Ce qui est alors requis, c'est une prise de position en valeur, la mobilisation d'un point de vue fort. C'est la mise à l'épreuve de la subjectivité au travail : il faut simultanément se confronter aux raisons que l'on a soi-même de se trouver là, engagé pour faire ce que l'on nous demande (ce qui conditionne l'appréhension des objectifs fixés par l'organisation); se confronter aussi aux enjeux de ce que l'on s'apprête à faire (ce qui détermine la façon dont on envisage les résultats exigés) ; se confronter enfin aux risques que l'on est personnellement prêt à prendre, en termes de fatigue, de dommages, de réputation, etc. (ce qui influence la manière dont on va se saisir des moyens alloués).

Au début du dispositif donc, un discutant va inviter la personne volontaire à présenter son activité de travail, en structurant l'exposé en trois temps. C'est d'abord le repérage : ce terme fait référence à la première anticipation. Le narrateur décrit les différents aspects de son travail tel qu'il est modélisé, le rôle du discutant étant uniquement d'obtenir les précisions nécessaires à la 
bonne saisie du « scénario » de travail. Il s'agit alors de la situation-type. On cherchera à connaître les déterminants du travail : le cadre officiel y compris institutionnel, le service à rendre et ses bénéficiaires, l'organisation des équipes, la répartition des tâches, le découpage du temps, l'aménagement des espaces, les relations clients-fournisseurs... Ce sont toutes les contraintes structurelles qui sont exposées, le plus souvent à l'aide d'un schéma des locaux, lequel sera " habité » c'est-à-dire mentionnera les protagonistes habituels du lieu (notons que le terme de contrainte n'a ici aucune connotation a priori négative puisqu'il est seulement question d'inventorier ce que l'acteur doit prendre en compte).

Le deuxième temps, c'est l'ancrage. Il ne s'agit plus de la situation-type mais d'une situation-témoin : une véritable journée, relativement récente (dans un souci de qualité de la mémoire) et bien située et datée. Elle est toujours choisie par l'exposant, selon les critères qui lui semblent pertinents en lien avec ce qu'il veut donner à voir. Le rôle du discutant consiste cette fois à suivre de près le narrateur et à l'interrompre chaque fois qu'il commet une rupture dans la chronologie pour lui demander de compléter son récit. En effet, le seul repère objectif est ici le temps de l'horloge : à la manière d'une main courante dans l'escalier, le temps objectif devient un guide pour précisément permettre au temps subjec- tif de s'exprimer. En dehors de ce rappel chronologique, le discutant ne se permet aucun jugement pendant l'exposé.

Nous abordons maintenant la vie réelle, le moment du travail. Celui-ci n'est pas constitué d'une suite d'instants (comme le laisse croire un planning) mais de la superposition des temps : un passé qui persiste par ses effets, un présent fait de simultanéités, un futur qui pèse déjà dans l'actuel. C'est pourquoi le narrateur est tenu de suivre la règle d'or dont nous venons de parler, le respect minutieux de la chronologie. Selon la séquence qu'il a choisie, il doit se tenir à la stricte succession des événements, justement parce que les phénomènes ne peuvent être appréhendés que dans l'ordre de leur apparition. C'est aussi une condition pour saisir le sens des micro-arbitrages des protagonistes. Enfin dès lors que l'on cherche à saisir des traces de la subjectivité au travail en dépliant les triangles agir-savoirs-valeurs, il nous faut raisonner sur le travail de quelqu'un de bien identifié : le narrateur par conséquent distinguera " les autres et moi ", en parlant de lui-même dans sa relation aux autres - et non en se plaçant à l'échelle d'un collectif. Toutefois il commencera systématiquement son récit par les autres, car la compréhension ultérieure de la moindre de ses décisions in situ en dépend : au moment d'agir, il a dû en effet toujours tenir compte des initiatives d'autrui dans le cours de 
l'action - des initiatives qui du coup sont devenues des contraintes pour lui-même.

Nous en arrivons au troisième temps, que nous appelons l'arbitrage. De quoi disposons-nous à ce moment-là ? Nous avons engrangé beaucoup d'informations sur une même situation de travail, il convient à présent d'y reconnaître un ordre. La première série réunit les données de repérage : il s'agit de la situation de travail telle qu'elle est anticipée, pensée à l'avance par l'organisation. Nous avons une vue sur les normes antécédentes, autrement dit sur le prescrit mais bien plus encore. On nous a aussi fourni des éléments de compréhension de la structure où se déroule le travail : d'où elle vient (bribes d'histoire), ce qu'elle cherche à faire (bribes de projet d'entreprise), comment elle est organisée (agencement des équipes, des fonctions, des tâches).

La deuxième série collecte les données d'ancrage : ce sont des informations non pas redondantes mais au contraire nettement différentes du repérage, contrairement à ce que l'on imaginerait à distance. Certes le récit de la journée bien réelle va reprendre comme des pointillés les repères de la journée-type, mais il fourmille en réalité d'informations inédites. Pour garder la métaphore du cinéma, la différence entre le repérage et l'ancrage est du même ordre que celle entre le scénario et le film lui-même. Le moment de l'ancrage voit les contraintes se démultiplier, alors qu'elles n'ont été que schématisées par le repérage : ce sont d'abord celles qui étaient annoncées dans le scénario bien sûr, mais elles se complexifient en entrant dans une histoire bien réelle, avec des gens qui ne cessent de prendre des initiatives à leur niveau et qui par conséquent n'arrêtent pas de reconfigurer la scène où doit intervenir « le héros » du récit.

Ces deux séries d'informations nous ont renseignés sur la situation en termes de «faits " : la situation anticipée d'une part et la situation actualisée d'autre part. Il nous reste un aspect à découvrir : la situation subjectivement vécue, c'està-dire telle qu'elle a été interprétée par le sujet lui-même. On tient là l'objectif de notre troisième temps, l'arbitrage. Le discutant va pouvoir proposer au narrateur de donner son avis sur la confrontation entre le travail prévisionnel et le travail actuel : il apparaît forcément un écart à chacun des moments de l'exposé. La raison de cet écart ne tient pas à la manière dont la situation a été organisée (en tout cas pas nécessairement). Soutenir l'hypothèse que la réalisation pourrait être un simple décalque de la prévision (parce que celle-ci aurait été bien conçue), c'est confondre la vie et le concept, le registre de la réalité concrète et celui du discours forcément en abstraction. Ce type d'écart est en réalité consubstantiel à l'expérience humaine, la plupart du temps ce n'est pas « la 
faute de l'organisation ». En revanche, il sera hautement formateur pour l'exposant (c'est-à-dire l'auteur du récit d'activité) de mettre en mots sa propre interprétation de l'écart, à chaque étape de l'aventure qu'il raconte. Ce faisant, il va vérifier non seulement quels sont les savoirs qu'il a su mobiliser au travail mais surtout au nom de quel point de vue il a évalué la situation hic et nunc; au nom de quelles valeurs il a tranché, ce qui veut dire décider d'agir plutôt de telle façon que de telle autre, en fonction de l'importance relative qu'il accorde à telle chose ou à tel fait. Et la verbalisation du point de vue qui le guide dans l'action offrira au narrateur une nouvelle opportunité, celle de le partager avec les autres : en s'ouvrant au débat avec celles et ceux qui écoutent l'exposé et qui pourront enrichir et affiner sa réflexion.

\section{Une deuxième phase du dispositif pour dire le travail}

La première phase de notre dispositif « construire son récit d'activité » s'arrête là. Le défi a été d'atteindre, au-delà de la situation anticipée et modélisée dans le langage formel, la situation d'une part actualisée (les contraintes de l'ici-maintenant qui provoquent l'écart avec la prévision) et d'autre part personnalisée (la gestion personnelle de cet écart, selon un point de vue de plus en plus consolidé, de plus en plus éprouvé) en accédant au langage de l'action. Nous disposons à présent d'un matériau qui donne accès à la subjectivité au travail, aux débats intérieurs à la personne qui a été convoquée pour faire quelque chose. Afin de satisfaire sa revendication existentielle de rester au centre de sa propre vie, de résister à l'usage de soi par les autres en affirmant un usage de soi par soi, cette personne va prendre en compte le problème pour lequel on la prie d'apporter une solution et elle le fera passer au crible de sa propre évaluation, jusqu'à transformer cette demande venue des autres en un problème pour soi, auquel on décide soi-même de répondre en mobilisant les ressources que l'on juge pertinentes.

Le dispositif ne peut toutefois en rester là, car le matériau issu de cette déconstruction de l'expérience vécue (une déconstruction au sens d'une analyse critique) se trouve à l'état de puzzle au terme de l'exercice " repérage, ancrage, arbitrage».

La deuxième phase du dispositif consistera en une reconstitution des pièces dispersées, qui en l'état donnent une impression de surabondance dans les détails, tout en offrant un accès très nouveau à l'activité de travail considérée. Y compris dans la bouche du travailleur lui-même qui est l'auteur de l'exposé, il n'est plus question de dire : « ce n'est rien, ce qu'on a fait ; on devait 
le faire ; on n'a fait qu'appliquer le prescrit ». La preuve est maintenant faite que la situation de vie au travail n'est pas banale. Elle ne coïncide pas entièrement avec celle qui est imaginée en amont, même si l'organisateur pense avoir dit l'essentiel du travail à faire parce qu'il dispose de solides arguments adossés aux concepts des sciences et techniques. Or cette divergence, ou en tout cas ce dépassement du concept par la vie justifie amplement une intervention humaine, un génie humain - génie du kairos (ou saisie ingénieuse des occasions) faisant écho du côté de la réalisation au génie du concepteur des situations anticipées. On ne peut pas se passer du travailleur, même quand on croit qu'il n'a rien à dire d'original...

Comment s'y prendre pour reconstruire un discours à partir du matériau riche et désormais disponible, constitué non seulement de faits mais de débats du sujet dans l'action, permettant de rendre intelligibles au moins partiellement les décisions prises dans l'action et l'orientation donnée au cours des événements ? Rappelons que notre démarche s'inscrit dans le contexte de la formation professionnelle. Nous cherchons par conséquent non seulement à produire un discours communicable sur le travail, mais aussi à puiser dans ce récit des indices (des preuves tirées des faits et de l'expérience interprétée par l'auteur du récit) pour positionner l'apprenti se- lon un référentiel professionnel dans le cadre d'une approche par compétences.

Le récit d'activité doit devenir une production en soi, un exercice de synthèse qui restitue aussi bien les aspects objectifs que subjectifs du vécu en situation professionnelle. On s'inspirera du schéma narratif pour fabriquer l'histoire en termes communicables. La démarche est la suivante :

- Identifier les phases-clés du processus de travail accompli, en partant du matériau brut recueilli lors de l'exercice " repérage, ancrage, arbitrage ";

- Rapprocher ces phases-clés des différentes étapes d'un schéma narratif, afin de vérifier qu'il y a bien une dynamique de récit à construire à partir de ce qui a été exposé. Rappelons que chaque séquence de la vie au travail dont on cherche à rendre compte suppose un lieu, des protagonistes, une action, une temporalité.

- Reconstituer le récit selon la logique du schéma narratif : (a) la situation initiale (cadre de l'intrigue) ; (b) l'élément déclencheur (modificateur) ; (c) le déroulement (péripéties) ; (d) le dénouement (résolution).

La dernière étape consiste à faire le lien entre le contenu du récit et le contenu d'un référentiel de métier : dans quelle mesure le récit très personnel 
du travail donne-t-il un éclairage sur les compétences du protagoniste ? On cherche à décoller du vécu singulier pour rejoindre le codifié du métier, atteindre les références formelles de la profession à partir d'une tranche de vie, celle d'un récit d'activité.

Voici notre démarche de rapprochement entre le récit d'activité et le référentiel d'emploi ou de formation :

- Dans un premier temps, nous passons le récit d'activité au filtre des attendus du référentiel : identifier dans le récit lui-même les éléments qui relèvent de la description de l'emploi/ métier ou du cahier des charges de la formation ; reconnaître dans ce même récit les actes professionnels effectivement posés par l'apprenti ; cerner enfin les raisons d'agir de ce dernier. On remarquera que ce filtre reproduit le triptyque repérage, ancrage, arbitrage.

- Dans un deuxième temps, nous partons du récit que nous venons de considérer selon les trois temps de l'agir professionnel modélisés par la compétence (les conditions ou pouvoir-agir ; les actes posés ou savoir-agir ; les raisons ou vouloir-agir) pour le rapprocher des items de compétence énoncés dans le référentiel de la formation.
- Dans un troisième temps, nous analysons le processus de professionnalisation à l'œuvre : ou comment le novice produit du savoir sur les situations rencontrées (il se trouve par exemple certaines ficelles de métier) tout en renforçant son point de vue de professionnel (il fait des choix, assume des risques, prend des responsabilités).

\section{Conclusion}

Dans le cadre du présent article, nous n'avons pas la place d'illustrer notre propos par des exemples de récits d'activité. Nous allons cependant lister ce qu'apportent concrètement ces retours d'expérience menés selon une approche ergologique (ce qui veut dire : restitution de la "deuxième anticipation " avec le registre de la vie mis en regard du registre des concepts).

La méthode repérage/ancrage permet essentiellement à l'apprenti de comprendre le travail. Autrement dit : à quelles conditions on agit. On n'attend pas de tout maîtriser (de n'avoir plus aucune contrainte devant soi, aucun empêchement à la prise d'initiative / mythe de la toute-puissance). On sait aussi qu'on maîtrise toujours quelque chose (on dispose toujours des interstices pour agir / on n'est jamais totalement privé de marge de manœuvre, dans l'impuis- 
sance totale). On découvre qu'on peut développer sa puissance normative (l'initiative rebondit face aux contraintes, et ces dernières peuvent apparaître comme des opportunités, être tournées vers le projet d'agir).

Plus précisément : on agit toujours dans un espace contraignant (l'espace au sens matériel et humain ; l'espace normatif, l'espace historique, l'espace habité, l'espace symbolisé...Proximité et éloignement, présence et absence...) ; on se situe toujours dans le temps, dans un moment complexe, celui de l'action : présence $d u$ passé ; présence du futur ; toujours dans le présent de l'action) ; on agit toujours en fonction des autres, grâce aux autres, malgré les autres (tension entre usage de soi par les autres et usage de soi par soi); on n'applique pas une consigne, on la repense (on repense le problème pour soi ; on n'est pas soi-même une ressource, mais on a des ressources); on commence toujours par évaluer pour agir et on évalue le moment au présent (en fonction de cette évaluation, nous mobilisons des ressources, celles dont nous disposons et qui nous paraissent pertinentes); on ne se contente pas de mobiliser des savoirs formalisés (on dispose aussi de son expérience qui fait savoir ou prise sur le milieu); en travaillant, on est toujours à l'épreuve de l'incertitude (même si la procédure a tracé le chemin, on est pris dans des débats de normes, débats sur les manières de s'y prendre et sur les va- leurs sous-jacentes) ; on prend toujours un risque, celui de se tromper, de mal faire, car agir c'est choisir, c'est trancher (on renormalise car il est impossible d'adopter deux manières de faire simultanément); on a un point de vue qui nous guide dans l'action, dans le discernement (un point de vue qui garantit la cohérence dont on a besoin pour agir mais en même temps qui est en devenir et non figé, qui se déplace... Et plus notre point de vue se confronte à celui des autres, plus il est fort, plus il nous fait exister socialement).

L'apprenti se découvre finalement digne du jugement de compétence par les autres quand il se découvre lui-même comme porteur d'un point de vue, producteur d'un savoir sur une situation spécifique, producteur de normes pour son propre agir et en débat de normes avec les autres - créatif dans le respect des exigences préalables. Cela lui donne une vision riche de lui-même et lui procure de nouvelles raisons de poursuivre sa formation - par exemple, en se constituant un portfolio à partir de ses récits d'activité. 
Language and work: a dynamic of "double anticipation" for vocational training

\section{Abstract}

In vocational training, the question of feedback arises. What does the work teach us, not only in terms of the knowledge to be mobilized in action, but also in the management of hardships when acting, when we have to take into account what has just happened and what is coming, initiatives that others are taking, issues of collective action, risks that we must take responsibility ourselves? To account for all of this, it is necessary to understand why it is so difficult to "say one's work" and to imagine a device capable of encouraging everyone to practice. This is the double purpose of this article.

Keywords: Activity story. Professional training. Language and ergology. Analysis of the activity.

\section{Bibliographie}

BARTHES, R. Leçon. Paris: Seuil, 1978.

BERGSON, H. (1938). La pensée et le mouvant. Paris : PUF, coll. Quadrige, 2003.

. (1929). Matière et mémoire. Paris :

PUF, coll. Quadrige, 2013.

CANGUILHEM, G. Milieu et normes de l'homme au travail. Cahiers internationaux de sociologie, vol. III, 1947, p.120-135.

. (1966). Le normal et le pathologique.

Paris : PUF, coll. Quadrige, 2003.

. (1952). La connaissance de la vie.

Paris: Vrin, 2006.
FOUCAULT, M. L'ordre du discours. Paris : Gallimard, coll. NRF, 1971.

FAÏTA, D. Le langage comme activité (159181). In: SCHWARTZ, Y. ; DURRIVE, L. (Ed.). Travail et ergologie, entretiens sur l'activité humaine I. Toulouse : Octarès editions, 2003.

RICEUR, P. Temps et récit. Tome 1. Paris : Seuil, 1983.

SCHWARTZ, Y. ; DURRIVE, L. (Ed.). L'Activité en dialogues, entretiens sur l'activité $h u$ maine II. Toulouse : Octarès editions, 2009.

. Travail et philosophie, convocations mutuelles. Toulouse : Octarès editions, 1992.

. Le paradigme ergologique ou un métier de philosophe. Toulouse : Octarès editions, 2000.

Produire des savoirs entre adhérence et désadhérence. In: BÉGUIN, P. ; CERF, M. (Ed.). Dynamique des savoirs, dynamique des changements. Toulouse, France : Octarès. 2009. p. 15-28.

(1988). Expérience et connaissance du travail. Paris : Editions sociales, coll. Les essentielles, 2012.

. Ergonomie, philosophe et exterritorialité (127-164). In: DANIELLOU, F. (Ed.). L'ergonomie en quête de ses principes, débats épistémologiques. Toulouse : Octarès éditions, 2015. 